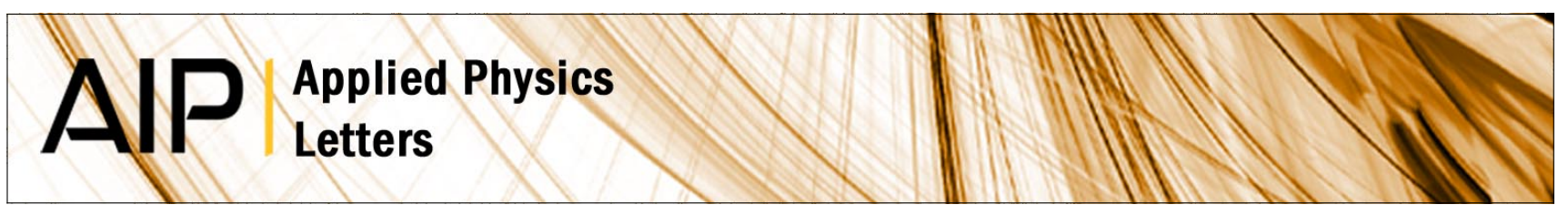

\title{
Thermal stress in silica-on-silicon disk resonators
}

Tong Chen, Hansuek Lee, and Kerry J. Vahala

Citation: Appl. Phys. Lett. 102, 031113 (2013); doi: 10.1063/1.4789370

View online: http://dx.doi.org/10.1063/1.4789370

View Table of Contents: http://apl.aip.org/resource/1/APPLAB/v102/i3

Published by the American Institute of Physics.

\section{Related Articles}

High-power microwave filters and frequency selective surfaces exploiting electromagnetic wave tunneling through -negative layers

J. Appl. Phys. 113, 064909 (2013)

Pulsed-laser deposited $\mathrm{Pb}(\mathrm{ZrO}$.52,Ti0.48)O3-on-silicon resonators with high-stopband rejection using feedthrough cancellation

Appl. Phys. Lett. 102, 063509 (2013)

Novel microwave near-field sensors for material characterization, biology, and nanotechnology

J. Appl. Phys. 113, 063912 (2013)

Note: Tunable notch filter based on liquid crystal technology for microwave applications

Rev. Sci. Instrum. 84, 026102 (2013)

Active microwave absorber with the dual-ability of dividable modulation in absorbing intensity and frequency AlP Advances 3, 022114 (2013)

\section{Additional information on Appl. Phys. Lett.}

Journal Homepage: http://apl.aip.org/

Journal Information: http://apl.aip.org/about/about_the_journal

Top downloads: http://apl.aip.org/features/most_downloaded

Information for Authors: http://apl.aip.org/authors

\section{ADVERTISEMENT}

\section{AIP Applied Physics Letters}

\section{EXPLORE WHAT'S NEW IN APL}

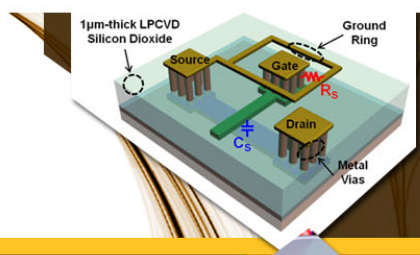

SURFACES AND INTERFACES

Focusing on physical, chemical, biological, structural, optical, magnetic and electrical properties of surfaces and interfaces, and more.. 


\title{
Thermal stress in silica-on-silicon disk resonators
}

\author{
Tong Chen, Hansuek Lee, and Kerry J. Vahala ${ }^{\text {a) }}$ \\ T. J. Watson Laboratory of Applied Physics, California Institute of Technology, Pasadena, California 91125 , \\ USA
}

(Received 4 December 2012; accepted 8 January 2013; published online 24 January 2013)

\begin{abstract}
The thermal expansion mismatch of thermal grown silica on a silicon wafer is well known to induce compressive stress upon cooling from the growth temperature to room temperature. In this Letter, we investigate how this stress impacts silica disk structures by comparison of measurements with both a finite element and an analytical model. The disk structures studied are also whispering gallery optical resonators, and proper control of stress is critical to obtain high-Q resonances. Based on our analysis, thicker oxide layers and proper control of undercut enable ultra-high-Q optical performance and mechanical stability. (C) 2013 American Institute of Physics. [http://dx.doi.org/10.1063/1.4789370]
\end{abstract}

For the past few decades, numerous experimental and theoretical studies have considered stress behavior and its impact on devices. ${ }^{1-3}$ In the silicon system, the oxide silica is grown at temperatures near $1000^{\circ} \mathrm{C}$. Upon cooling to room temperature, the difference between the thermal expansion coefficients of the silica and silicon causes a well-known compressive stress in the oxide. While this stress typically does not cause yield, reliability, or performance issues in photonic devices, certain whispering gallery style optical resonators and waveguides rely upon undercut of the oxide to create optical confinement. ${ }^{4-6}$ The undercut silica layer creates an aircladding to guide the light (Fig. 1(a)), and if there is sufficient thermal-induced stress, then this thin air-cladding silica layer may buckle and form crown-like patterns. Understanding the buckling behavior is important in optimizing the device performance. Specifically, oxide undercuts must be deep enough in these structures to isolate the optical mode from the silicon support pillar, however, the crowning behavior can occur at a critical undercut value and thereby interfere with optical performance. Likewise, in MEMS systems with free standing structures, residual stress can physically warp devices to a degree that renders them no longer useful. ${ }^{7,8}$ In this work, we measure the buckling behavior of silica disks structures and compare with two models so as to create design guidelines that eliminate buckling in both resonator and waveguide structures. Specifically, by proper selection of oxide thickness and undercut, excellent optical performance is obtained (over 800 million optical quality factor for disk resonator structures of $7.5 \mathrm{~mm}$ diameter and $10 \mu \mathrm{m}$ oxide thickness ${ }^{4}$ ).

Earlier work on silica wedge-shaped resonators achieved Q factors as high as 50 million ${ }^{9}$ in devices with diameters around $100 \mu \mathrm{m}$. Those structures featured a lithographically defined oxide disk of $2 \mu \mathrm{m}$ thickness that had been partially undercut using the silicon selective etchant xenon diflouride. The ability to extend both the $\mathrm{Q}$ factor and the resonator diameter to larger values (Q greater than 100 million and resonator diameter to the $\mathrm{mm}-\mathrm{cm}$ size range) is important for applications such as microcombs and rotation sensing. ${ }^{10,11}$ However, due to thermal stress, simply scaling the previous device to larger diameters fails to provide satisfactory

\footnotetext{
${ }^{\text {a) }}$ Author to whom correspondence should be addressed. Electronic mail: vahala@caltech.edu.
}

performance. Figure 1(b) shows a top view (interference contrast mode) image of a silica wedge resonator having $500 \mu \mathrm{m}$ diameter and $2 \mu \mathrm{m}$ oxide thickness. With less than $70 \mu \mathrm{m}$ undercutting of the silicon, the silica layer starts to buckle and features a crown-like pattern. Further, the number of nodes in the buckled pattern decreases with the deepness of the undercut. For example, as shown in Figs. 1(b)-1(e), the number of nodes in the buckled structures are 18, 10, 8 , and 6 for $70 \mu \mathrm{m}, 120 \mu \mathrm{m}, 155 \mu \mathrm{m}$, and $180 \mu \mathrm{m}$ undercuts. Ultimately, if the undercut is deep enough, the resonator will return to the unbuckled configuration (Fig. 1(f)).

To assess the impact of buckling on the optical performance of the resonators, the intrinsic optical $Q$ factor ${ }^{12}$ of normal and buckled samples was measured. Devices were coupled to an optical fibre using a fibre taper, ${ }^{13,14}$ and spectral lineshape data were obtained by tuning an external cavity semiconductor laser $(1550 \mathrm{~nm})$ across an optical resonance while monitoring transmission on an oscilloscope (see sample scan in Fig. 1(g)). To accurately calibrate the laser scan in this measurement, a portion of the laser output was also monitored after transmission through a calibrated Mach-Zehnder interferometer having a free-spectral-range of $6.72 \mathrm{MHz}$. The buckled resonators (Figs. 1(b)-1(e)) show low Q factors, typically below 1 million. Moreover, some of these samples were observed to crack over a period of a few days. In contrast, an unbuckled $500 \mu \mathrm{m}$ diameter resonator with $2 \mu \mathrm{m}$ thickness (corresponding to an undercut of $55 \mu \mathrm{m}$ in Fig. 1(a)) featured a 38 million Q factor, and this Q-factor performance was preserved for more than 1 year. The spectral scan for this resonator is shown in Fig. 1(g).

To model thermal stress, we take the difference between oxide growth temperature $\left(1000^{\circ} \mathrm{C}\right)$ and the simulation temperature as a control parameter $(\Delta T)$ and simulate the equilibrium state of the resonator via a finite element model in COMSOL MULTIPHYSICS (http://www.comsol.com/). The following is defined as an order parameter

$$
\Omega=\frac{1}{V} \iiint_{\text {silica }} \sqrt{\left(\frac{\partial w}{\partial x}\right)^{2}+\left(\frac{\partial w}{\partial y}\right)^{2}} d V
$$

where $w$ is the vertical component of deformation due to thermal stress, $V$ is the volume of silica, and integration is carried over the whole silica layer. The order parameter is a simple 

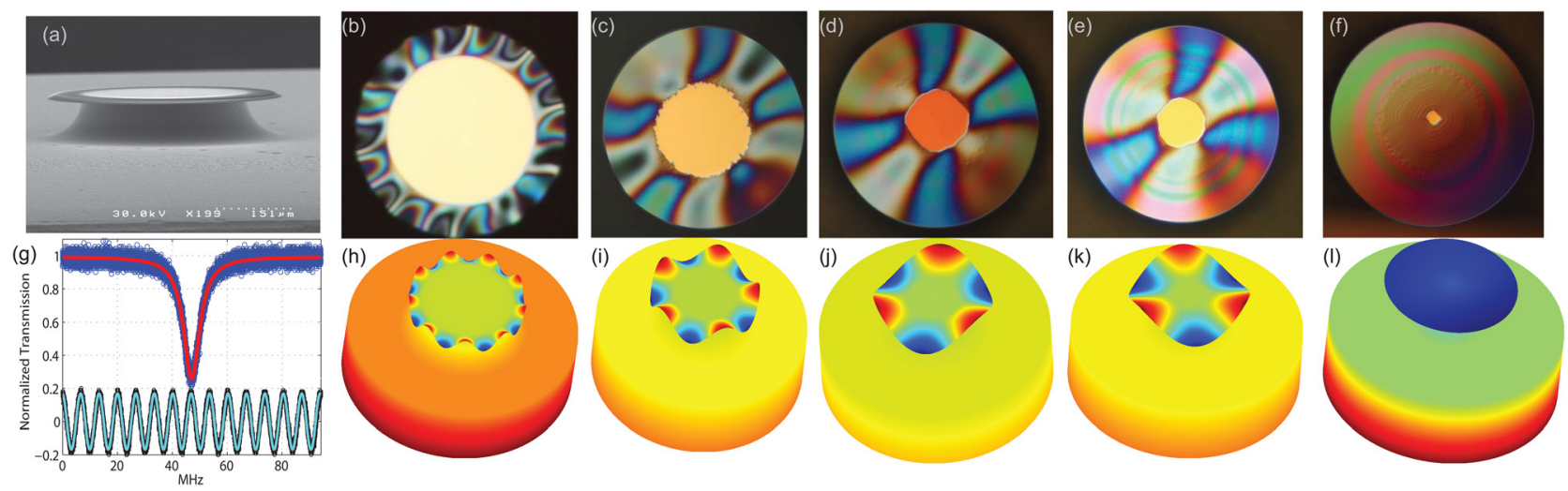

FIG. 1. (a) SEM image of an unbuckled silica disk ( $500 \mu \mathrm{m}$ diameter and $2 \mu \mathrm{m}$ oxide thickness) resonator on a silicon pillar. The undercut is about $55 \mu \mathrm{m}$. (b)(f) Top-view microscope images of resonators ( $500 \mu \mathrm{m}$ diameter and $2 \mu \mathrm{m}$ oxide thickness) with different buckled oxide configurations. The undercuts of these devices are $70 \mu \mathrm{m}, 120 \mu \mathrm{m}, 155 \mu \mathrm{m}, 185 \mu \mathrm{m}$, and $225 \mu \mathrm{m}$ respectively. (g) Spectral scan for the resonator in panel (a). The measured linewidth corresponds to an optical Q factor of 37 million. The red curve gives a Lorentzian fitting of the experimental transmission and the cyan line shows a sinusoidal fitting of the interferometer output. (h)-(i) Finite element simulation results of resonators (500 $\mu \mathrm{m}$ diameter and $2 \mu \mathrm{m}$ oxide thickness) showing different buckling configurations. The undercut of these devices match those in the panels (b)-(f).

measure of the irregularity of the silica induced by the stress. Figure 2 is a plot of the numerically simulated order parameter plotted versus $\Delta T$. The plot shows a second-order phase transition behavior as $\Delta T$ increases in a resonator of diameter $500 \mu \mathrm{m}$, silica layer thickness $2 \mu \mathrm{m}$, and undercut of $70 \mu \mathrm{m}$. Above a critical temperature difference $\left(\Delta T_{c}\right)$, the silica layer buckles and features a crown-like pattern with 16 nodes. In contrast, the silica layer bends uniformly downward when $\Delta T$ is smaller than the critical value. Since the silica is grown at $100{ }^{\circ} \mathrm{C}$, the equilibrium state of this resonator at room temperature will have a buckled pattern.

The equilibria of resonators with differing amounts of undercut were also calculated for comparison with the measurements in Fig. 1 (see, Figs. 1(h) and 1(i)). Ultimately, if the undercut is deep enough, the silica layer will uniformly bend downward. The simulated configurations have 16,10 , 6, and 6 nodes for $70 \mu \mathrm{m}, 120 \mu \mathrm{m}, 155 \mu \mathrm{m}$, and $180 \mu \mathrm{m}$ undercuts and are more or less consistent with measurements. The discrepancy might result from the slight irregularity in the etched silicon pillar.

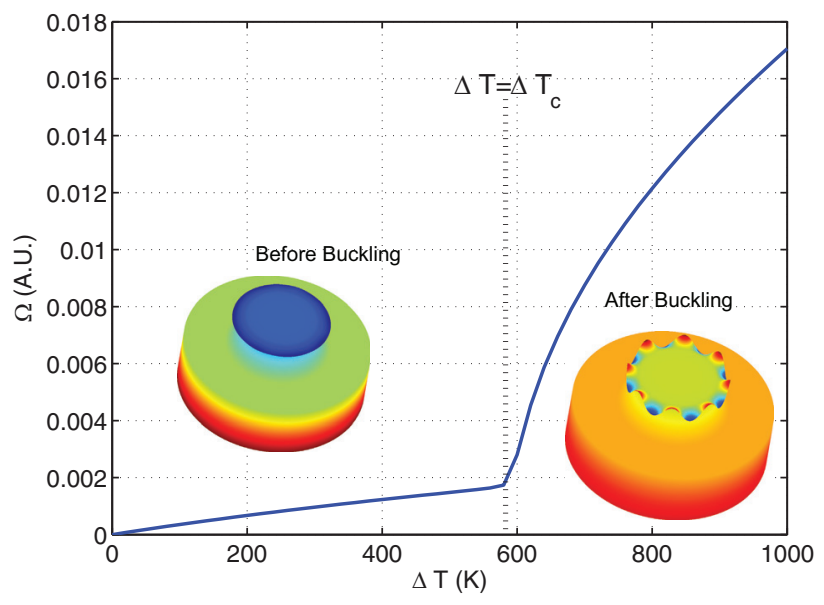

FIG. 2. Plot of the order parameter (Eq. (1)) versus the change in temperature relative to the growth temperature. A second-order phase transition is apparent at a critical temperature difference $\left(\Delta T_{c}\right)$. Above this value, the disk buckles and features a crown pattern with several nodes. On the other hand, the silica layer bends uniformly down when $\Delta T$ is smaller than the critical value. In this FEM simulation, the resonator has diameter $500 \mu \mathrm{m}$, thickness $2 \mu \mathrm{m}$, and undercut $70 \mu \mathrm{m}$.
In addition to the finite element analysis, an analytic model was studied to provide guidance on device design. The approach is based on a two-dimensional buckling model of the annular disk and the energy method. The energy model employed is especially useful when a rigorous solution of the Kirchkoff equation is unknown or it is required to find only an approximate value of the critical temperature difference for buckling. ${ }^{15-17}$ Basically, if the work done by thermal stress is smaller than the strain energy of bending for every possible shape of buckling, then the unbuckled equilibrium is stable. If the same work becomes larger than the energy of bending for any shape of deformation, then the structure tends to be unstable and starts buckling. In a silica disk resonator with radius $b$, thickness $t$, and for a silicon pillar of radius $a$, the buckling shape can be approximated by a sine curve along the circumference of a plate. ${ }^{18,19}$ Assume that the deflection of the annular disk in the vertical direction is

$$
w=C(r-a)^{2} \cos (n \theta),
$$

where $C$ is the amplitude of buckling, $2 n$ is the number of nodes in the crown-like pattern, and the deflection obeys the clamped boundary condition at $r=a$. With respect to a polar coordinate system $(r, \theta)$ with origin at the center, the components of stress induced by the thermal expansion mismatch are ${ }^{20}$

$$
\begin{gathered}
\sigma_{r}(r)=\frac{b^{2}-a^{2}}{a^{2}\left(\frac{b^{2}}{r^{2}}-1\right)} \sigma, \\
\sigma_{\theta}(r)=-\frac{b^{2}-a^{2}}{a^{2}\left(\frac{b^{2}}{r^{2}}+1\right)} \sigma,
\end{gathered}
$$

where

$$
\begin{gathered}
\sigma=\frac{\Delta \alpha \cdot \Delta T}{\frac{1-\nu_{S i}}{E_{S i}}+\frac{1}{E_{S i O_{2}}}\left(\frac{1+\rho^{2}}{1-\rho^{2}}\right)+\frac{\nu_{S i O 2}}{E_{S i O_{2}}}} \\
=\Delta \alpha \cdot \Delta T \cdot h(\rho)
\end{gathered}
$$

and where $\rho=a / b, \nu_{\mathrm{Si}}$ and $\nu_{\mathrm{SiO}_{2}}$ are the Young's moduli of silicon and silica; $E_{\mathrm{Si}}$ and $E_{\mathrm{SiO}_{2}}$ are the Poisson's ratio of silicon and silica; $\Delta \alpha$ is the difference of the thermal expansion 
coefficients; and $\Delta T$ is the temperature difference between oxidation growth temperature and room temperature. For clarity, we defined that $h(\rho)=\frac{1}{\frac{1-\nu_{S i}}{E_{S i}}+\frac{1}{E_{S_{i O}}}\left(\frac{1+\rho^{2}}{1-\rho^{2}}\right)+\frac{\nu_{S i O O}}{E_{S i O}}}$. The bending energy is ${ }^{15}\left(\nu=\nu_{\mathrm{SiO}_{2}}, D=\frac{E_{\mathrm{SiO}_{2}} t^{3}}{12\left(1-\nu_{\mathrm{SiO}_{2}}\right)}\right)$,

$$
\begin{aligned}
U= & \iint r d r d \theta\left[\frac{D}{2}\left(\frac{\partial^{2} w}{\partial r^{2}}+\frac{1}{r} \frac{\partial w}{\partial r}+\frac{1}{r^{2}} \frac{\partial^{2} w}{\partial \theta^{2}}\right)^{2}\right. \\
& -D(1-\nu) \frac{\partial^{2} w}{\partial r^{2}}\left(\frac{1}{r} \frac{\partial w}{\partial r}+\frac{1}{r^{2}} \frac{\partial^{2} w}{\partial \theta^{2}}\right) \\
& \left.+D(1-\nu)\left(\frac{1}{r} \frac{\partial^{2} w}{\partial r \partial \theta}-\frac{1}{r^{2}} \frac{\partial w}{\partial \theta}\right)^{2}\right] .
\end{aligned}
$$

The work done by the silica layer forces during the buckling is found to be $\mathrm{e}^{15}$

$$
T=-\frac{1}{2} \iint r d r d \theta\left[\sigma_{r} t\left(\frac{\partial w}{\partial r}\right)^{2}+\sigma_{\theta} t\left(\frac{1}{r} \frac{\partial w}{\partial \theta}\right)^{2}\right],
$$

where $t$ is the thickness of the silica layer. By equating $U$ and $T$, the critical condition of buckling can be written as

$$
\Delta T=\frac{2 t^{2} E_{\mathrm{SiO}_{2}}}{b^{2}\left(1-\nu_{\mathrm{SiO}_{2}}^{2}\right) \Delta \alpha h(\rho)} \cdot \frac{F(n, \rho)}{G(n, \rho)},
$$

where

$$
\begin{aligned}
F(n, \rho)= & (-1+\rho) \times[8(1+\nu)(-1+\rho) \\
& +2 n^{4}(-1+\nu)(1+\rho)(1+(-8+\rho) \rho) \\
& \left.-n^{2}(13+12 \nu(-1+3 \rho)+\rho(-43+(-7+\rho) \rho))\right] \\
& +4\left(-2+6 n^{4}(-1+\nu)+n^{2}(-9+6 \nu)\right) \rho^{2} \log (\rho),
\end{aligned}
$$

$$
\begin{aligned}
G(n, \rho)= & \frac{\rho^{2}}{-1+\rho^{2}} \times\left[4\left(3+\rho\left(-16+12 \rho+\rho^{3}\right)\right)\right. \\
& -n^{2}\left(9+\rho\left(-64+36 \rho+19 \rho^{3}\right)\right) \\
& \left.+12 \rho^{2}\left(-4+n^{2}\left(6+\rho^{2}\right)\right) \log (\rho)\right] .
\end{aligned}
$$

Figure 3 shows the relation between the normalized critical temperature difference $\left(\Delta \alpha \Delta T b^{2} / t^{2}\right)$ and parameter $\rho=a / b$ for configurations with different numbers of nodes in the silica/silicon disk system. The normalization used here provides a dimensionless parameter that characterizes the threshold of buckling. It also reflects the fact that critical temperature difference depends quadratically on the resonators' oxide thickness and inversely quadratically on their radius. At each $\rho$, the configuration with minimal critical temperature difference gives the equilibrium state after buckling. As the undercut goes deeper, the equilibrium buckled configuration will have fewer nodes. This result is consistent with both experimental observation and the finite element simulation. Further, the combination of these curves defines an envelope function that outlines the boundary between the unbuckled and buckled state (cf., Fig. 2). It also explains the fact that the resonator has an unbuckled configuration for small silicon pillar (Fig. 1(f)).

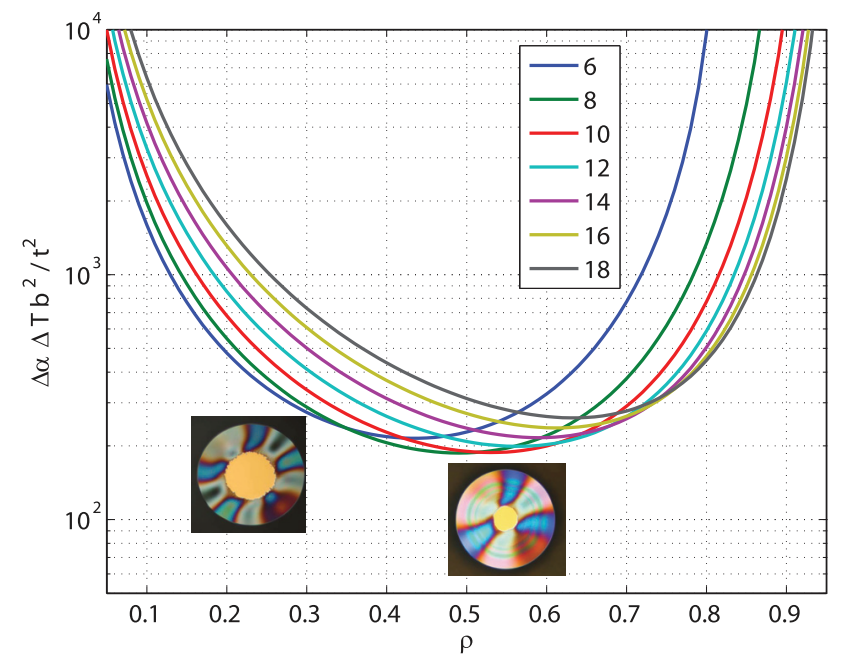

FIG. 3. The relation between normalized critical temperature difference $\left(\Delta \alpha \Delta T b^{2} / t^{2}\right)$ and parameter $\rho=a / b$ for configurations with different numbers of nodes (see legend) in the silica/silicon system. Insets show examples from experiments of the buckled configurations with $\rho=0.52,10$ nodes and $\rho=0.26,6$ nodes (see, Fig. 1).

The y-axis parameter in Fig. 3 shows that the critical temperature difference depends quadratically on the thickness of the silica layer. It implies that thicker oxides will avoid the buckling and maintain mechanical stability. Interestingly, optical performance, in particular the Q factor, also improves for thicker oxides. This can be understood to result because the optical field at the oxide-air interface is generally weaker for thicker oxide. ${ }^{4}$ This reduces both surface absorption and scattering, thereby increasing the optical Q factor. From a design perspective, the undercut needs to be carefully controlled. On one hand, it must be deep enough to reduce optical loss due to the mode leakage into the silicon pillar. On the other hand, shallow undercut is more desirable to avoid buckling. Indeed, we have demonstrated elsewhere ${ }^{4}$ that by increasing the oxide thickness to $10 \mu \mathrm{m}$ and controlling the undercut to be approximately $150 \mu \mathrm{m}$, it is possible to obtain a record $\mathrm{Q}$ factor on a chip of 875 million $\mathrm{Q}$ in a device that is mechanically stable.

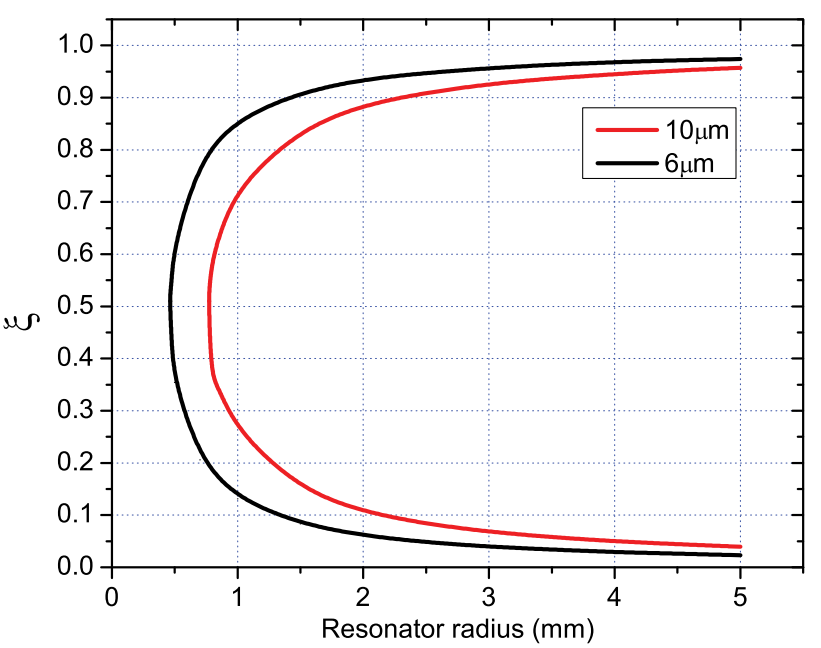

FIG. 4. The threshold of undercut parameter $(\xi=1-a / b)$ for buckling. For each thickness, the left side of curve defines a region in which the disk resonators do not buckle. 
To provide guidance to disk resonator design, we calculate the buckling threshold of undercut for different disk resonators having $6 \mu \mathrm{m}$ and $10 \mu \mathrm{m}$ oxide thickness. As shown in Figure 4, for each resonator radius there are two thresholds (lower and higher). If the undercut is smaller than the lower threshold or larger than the higher threshold, the resonator remains in the unbuckled configuration. In contrast, if the undercut is between these two thresholds, the resonator will buckle (cf., Fig. 1).

In summary, this work demonstrates and models the impact of the thermal stress in silica-on-silicon disk resonators. We provide both analytical and finite element modeling to understand buckling behavior and offer guidance on performance improvement. In particular, by proper design, stress does not limit the optical performance of these devices. Although we only discuss the particular case of the disk resonator, our analysis could be extended to guide the design of other structures.

We gratefully acknowledge the Defense Advanced Research Projects Agency under the iPhoD program, the Institute for Quantum Information and Matter, an NSF Physics Frontiers Center with support of the Gordon and Betty Moore Foundation, and also the Kavli Nanoscience Institute at Caltech. H.L. thanks the Center for the Physics of Information.
${ }^{1}$ N. J. Hoff, J. R. Aeronaut. Soc. 61, 756-774 (1957).

${ }^{2}$ E. I. Grigolyuk and L. A. Magerramova, Izv An SSSR. Mekh. Tverd. Tela 16, 111-138 (1981)

${ }^{3}$ T. R. Tauchert, Appl. Mech. Rev. 44, 347-360 (1991).

${ }^{4}$ H. Lee, T. Chen, J. Li, K. Yang, S. Jeon, O. Painter, and K. J. Vahala, Nature Photon. 6, 369-373 (2012).

${ }^{5}$ H. Lee, T. Chen, J. Li, O. Painter, and K. J. Vahala, Nat. Commun. 3, 867 (2012).

${ }^{6}$ D. K. Armani, T. J. Kippenberg, S. M. Spillane, and K. J. Vahala, Nature 421, 925-929 (2003).

${ }^{7}$ L. Starman, J. Busbee, J. Reber, J. Lott, W. Cowan, and N. Vandelli, Nanotechnology 1, 398-401 (2001).

${ }^{8}$ W. Spengen, Microelectron. Reliab. 43, 1049-1060 (2003).

${ }^{9}$ T. J. Kippenberg, J. Kalkman, A. Polman, and K. J. Vahala, Phys. Rev. A 74, 051802 (2006).

${ }^{10}$ T. J. Kippenberg, R. Holzwarth, and S. A. Diddams, Science 332, 555-559 (2011).

${ }^{11}$ C. Ciminelli, F. Dell'Olio, C. Campanella, and M. Armenise, Adv. Opt. Photon. 2, 370-404 (2010).

${ }^{12}$ K. J. Vahala, Nature 424, 839-846 (2003).

${ }^{13}$ M. Cai, O. J. Painter, and K. J. Vahala, Phys. Rev. Lett. 85, 74 (2006).

${ }^{14}$ S. M. Spillane, T. J. Kippenberg, O. J. Painter, and K. J. Vahala, Phys. Rev. Lett. 91, 043902 (2003).

${ }^{15}$ S. P. Timoshenko and J. Gere, "Theory of Elastic Stability" (McGraw-Hill Book Company, 1961).

${ }^{16}$ E. H. Mansfield, J. Mech. Appl. Math. 13, 16-23 (1960).

${ }^{17}$ K. K. Raju and G. V. Rao, Comput. Struct. 18, 1179-1182 (1984).

${ }^{18}$ T. X. Yu and W. Johnson, Int. J. Mech. Sci. 24, 175-188 (1982).

${ }^{19}$ C. Y. Wang, J. Appl. Mech. 72, 795-796 (2005).

${ }^{20}$ T. Tang, C. Y. Hui, H. G. Retsos, and E. J. Kramer, Eng. Fract. Mech. 72, 791-805 (2005). 